\title{
Ultrasonic Study of Molecular Association of Ternary Mixtures in Polyvinyl Alcohol, Stearates and Ethylene Glycol
}

\author{
${ }^{1}$ R. Kavitha , ${ }^{2}$ S. Jayakumar and ${ }^{3}$ R. Uma \\ ${ }^{1}$. Department of Chemistry, Easwari Engineering College, Chennai -89. \\ 2. Department of Physics, Vivekananda college, Mylapore, Chennai - 04. \\ 3. Department of Chemistry, Pachaiyappa's College, Chetpat, Chennai-30.
}

\begin{abstract}
Ultrasonic velocity measurements are reported for aqueous solutions of polyvinyl alcohol with different stearates such as zinc stearate and calcium stearate with plasticizer ethylene glycol at $303 \mathrm{~K}$. The data obtained as a function of concentration indicate the magnitude of the contributions due to the relaxation of the backbone and of the side - chain acetyl group. A variety of the different theoretical models has been proposed to attempt to explain the way in which the factors such as frequency, intensity, solvent, temperature, nature of dissolved gas, external pressure and the molecular mass distribution influence the rate of degradation, and final molecular mass of the degraded species.
\end{abstract}

Keywords: Polyvinyl alcohol, Glycol, Stearate, Stabilizer, Plasticizer

\section{Introduction}

Ultrasonic propagation has traditionally been used as a test for high frequency dynamics. Using ultrasonic waves, it is possible to sense the molecular structure and motion in liquid polymer systems. The ultrasonic attenuation $\alpha$ is noticeably influenced by the chemical structure and can be associated with conformational changes of elements of the polymer chain of varying size. The past few decades have seen a dramatic rise in the applications of high - intensity, or power, ultrasound in chemistry, with a range of synthetic procedure and process methods having found to benefit. One of the beneficial applications of ultrasound is the polymer degradation especially considering the fact that there are no changes in the chemical nature of polymer and the reduction in molecular weight is simply by splitting the most susceptible chemical bond [1]. The study of molecular interactions in the polymer and solvent throws light on the processes involving polymer production and their uses [2]. In general, it can be said that ultrasonic irradiation does have a significant effect on polymers in terms of its mechanical, mechano - chemical and morphological properties. Many of the investigation have undertaken these studies qualitatively through ultrasonic velocity, density, adiabatic compressibility and viscometric data. The values of ultrasonic velocity $(\mathrm{U})$, density $(\rho)$ and viscosity $(\eta)$ for the pure components is given in Table 1. From the experimental values, a few acoustical parameters such as adiabatic compressibility $(B)$, acoustical impedance $(\mathrm{Z})$, molar sound velocity $(\mathrm{R})$, Wada's constant $(\mathrm{W})$, molar volume $(\mathrm{Vm})$, free volume $(\mathrm{Vf})$, intermolecular free length (Lf), internal pressure $(\pi)$, absorption coefficient $(\alpha / \mathrm{f} 2)$ viscous relaxation time ( 1 ), degree of intermolecular attraction ( $\alpha$ ), excess ultrasonic velocity (UE), excess adiabatic compressibility $(ß \mathrm{E})$, excess acoustical impedance (ZE), excess free length (LfE) and excess molar volume (VmE) were derived over the entire mole fraction range. Ultrasonic velocities have also been evaluated theoretically with the help of Impedance relation, Nomoto relation, Van Dael \& Vangeel relation and Junjie relation. The suitability of these theories and equations were checked by comparing theoretical values of ultrasonic speeds with the values obtained experimentally. Literature survey showed that no measurements have been previously reported for the mixtures reported in this paper.

\section{Materials And Methods}

The chemicals used were of analytical grade and obtained from E.Merck company. Thermostatically controlled well-stirred water bath whose temperature was maintained to $\pm 0.01 \mathrm{~K}$ accuracy was used for all the measurements. Binary mixtures were prepared by weighing in airtight bottles, the possible uncertainty in the concentration is estimated to be less than \pm 0.0001 . Densities of pure components and their mixtures were determined by using a $1 \times 10-5 \mathrm{~m} 3$ double arm pycnometer. The density values from triplicate replication at the temperature of $303 \mathrm{~K}$ were reproducible within $\pm 2 \times 10-2 \mathrm{~kg} \mathrm{~m}-3$. The uncertainty in density and excess molar volume values were found to be $\pm 4 \times 10-2 \mathrm{~kg} \mathrm{~m}-3$ and $\pm 0.001 \times 10-6 \mathrm{~m} 3$ mol-1 respectively. Ostwald's viscometer having capacity of about $15 \mathrm{ml}$ and the capillary having a length of about $90 \mathrm{~mm}$ and $0.5 \mathrm{~mm}$ internal diameter has been used to measure the flow times of pure liquids and liquid mixtures and it was calibrated with benzene (density $\approx 0.8738 \mathrm{~g} \mathrm{~cm}-3$ ) and doubly distilled water (density $\approx 0.9970 \mathrm{~g} \mathrm{~cm}-3$ ) at 303 $\mathrm{K}$. The flow time of pure liquids and liquid mixtures were repeated for five times. The uncertainty of viscosity 
was $\pm 0.005 \times 10-3 \mathrm{~m}$ Pas. Speed of sound was measured by using a variable path, single crystal interferometer. (United scientific company, India), working at $2 \mathrm{MHz}$ frequency. The interferometer was calibrated using toluene. Measurement of speed of sound through medium was based on the accurate determination of the wavelength of ultrasonic waves of known frequency produced by quartz crystal in the measuring cell. The interferometer cell was filled with the test liquid, and water was circulated around the measuring cell from a thermostat. The uncertainty was estimated to be $0.1 \mathrm{~ms}-1$.

The adiabatic compressibility (Bs) was calculated by the equation

$\beta=1 / \rho \mathrm{U} 2$

Where $\rho$ is the density of mixture and $\mathrm{U}$ is the ultrasonic velocity of the mixture.

The acoustical impedance $(Z)$ was calculated by the equation,

$\mathrm{Z}=\rho \mathrm{U}$

Where $\rho$ is the density of mixture and $U$ is the ultrasonic velocity of the mixture.

The molar sound velocity $(\mathrm{R})$ was calculated by the equation

$\mathrm{R}=($ Meff $/ \rho) \mathrm{U} 1 / 3$

Where $\mathrm{U}$ is the ultrasonic velocity of the mixture.

The molar compressibility or Wada's constant (W), was calculated by the equation

$\mathrm{W}=(\mathrm{M} / \rho) \beta-1 / 7$

Where $\mathrm{M}$ is the relative molar mass and $\beta$ is the adiabatic compressibility.

The intermolecular free length (Lf) was calculated by the equation

$\mathrm{Lf}=\mathrm{k} \beta 1 / 2$

Where $\mathrm{K}=1.98$ X 10-6, the Jacobson constant (Jacobson 1952).

The Free volume was calculated by the equation

$\mathrm{Vf}=(\mathrm{Meff} \mathrm{U} / \mathrm{K} \eta) 3 / 2$

Where $\mathrm{K}=4.28 \mathrm{X} 109$ for all liquids which is a temperature independent constant.

The internal pressure was calculated by the equation

$\pi=\{\mathrm{bRT} /(\mathrm{V} 2 \mathrm{Vf}) 1 / 3\}$

$\mathrm{b}$ is a packing factor, $\mathrm{R}$ is a gas constant, $\mathrm{Vf}$ is free volume and $\mathrm{T}$ is temperature.

The absorption coefficient was calculated by the equation

$(\alpha / \mathrm{f} 2)=(8 \pi 2 \eta / 3 \rho \mathrm{U} 3)$

$\eta$ is viscosity of the mixture and $\rho$ is the density of the mixture.

The viscous relaxation time was calculated by the equation

$\imath=(4 \eta / 3 \rho U 2)$

$\eta$ is viscosity of the mixture and $\rho$ is the density of the mixture.

The degree of intermolecular attraction $(\alpha)$ was calculated by the equation

$\alpha=(\mathrm{u} 2 / \mathrm{u} 2 \mathrm{im})-1$

Where $\mathrm{u} 2 \mathrm{im}=1 /\{(\mathrm{x} 1 \mathrm{M} 1+\mathrm{x} 2 \mathrm{M} 2)(\mathrm{x} 1 / \mathrm{M} 1 \mathrm{u} 12+\mathrm{x} 2 / \mathrm{M} 2 \mathrm{u} 22)\}$

The UE, $B \mathrm{E}, \mathrm{ZE}$, LfE, and $\mathrm{VmE}$ were derived over the entire mole fraction range by using the general equation $\mathrm{AE}=\mathrm{A}-(\mathrm{Xi} \mathrm{A} 1+(1-\mathrm{Xi}) \mathrm{A} 2)$

Where $\mathrm{A}$ is the corresponding parameters $(\mathrm{U}, \mathrm{B}, \mathrm{Z}, \mathrm{Lf}$, and $\mathrm{Vm})$ of mixxture and $\mathrm{A} 1$ and $\mathrm{A} 2$ are the corresponding pure component values.

The percentage deviation of the experimental velocity from the theoretical value is given by the equation

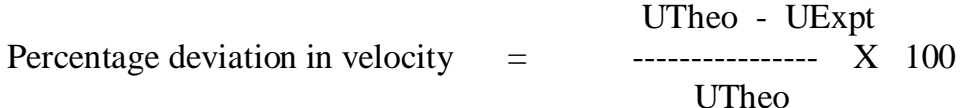

\section{Results And Discussion}

The ultrasonic velocity, density and viscosity data for the pure components at $303 \mathrm{~K}$ are given below: Table 1 Comparison of density, ultrasonic velocity and viscosity data at $303 \mathrm{~K}$

\begin{tabular}{|l|l|l|l|}
\hline Component & $\mathrm{U}$ & $\rho$ & $\eta \mathrm{X} 10-1$ \\
$\mathrm{~m} / \mathrm{s}$ & $\mathrm{Kg} / \mathrm{m} 3$ & $\mathrm{Nsm}-2$ \\
\hline $0.2 \%$ PVA & 1492 & 1100 & 1.05 \\
\hline Zinc Stearate & 1404 & 1133 & - \\
\hline Calcium Stearate & 1310 & 1145 & - \\
\hline Ethylene glycol & 1638 & 960 & 1.38 \\
\hline
\end{tabular}

Table 2 gives the measured and acoustic parameters such as ultrasonic velocities $(U)$, density $(\rho)$, viscosity $(\eta)$, adiabatic compressibility (ß), acoustical impedence (Z), molar sound velocity (R), molar compressibility (W), molar volume $(\mathrm{Vm})$, free volume $(\mathrm{Vf})$, Table 3 gives the thermodynamic properties like intermolecular free 
length (Lf), internal pressure $(\pi)$, absorption coefficient $(\alpha / \mathrm{f} 2)$, viscous relaxation time $(\mathfrak{l})$, degree of intermolecular attraction $(\alpha)$, Table 4 gives the excess parameters like excess ultrasonic velocity (UE), excess adiabatic compressibility (BE), excess acoustical impedance (ZE), excess free length (LfE), excess molar volume (VmE), Table 5 gives the theoretical values of ultrasonic velocity calculated from Impedance, Nomoto, Van Dael \& Vangeel and Junjie's relation along with the experimental ultrasonic velocity and percentage deviation for the ternary mixtures $0.2 \%$ PVA - Zn. St. - EG and $0.2 \%$ PVA - Ca. St. - EG over the entire composition range at $303 \mathrm{~K}$.

Table 2 : Measured and acoustic parameters of ternary mixtures at $303 \mathrm{~K}$

\begin{tabular}{|c|c|c|c|c|c|c|c|c|c|}
\hline $\begin{array}{l}\text { Conc } \\
\text { of stearate }\end{array}$ & $\begin{array}{l}\mathrm{U} \\
\mathrm{ms}-1\end{array}$ & $\begin{array}{l}\rho \\
\text { Kgm-3 }\end{array}$ & $\begin{array}{l}\eta / \\
\text { Nsm-2 }\end{array}$ & $\begin{array}{l}\beta / 10-10 \\
\text { Kg-1ms-2 }\end{array}$ & $\begin{array}{l}\mathrm{Z} / 106 \\
\mathrm{Kg} \text { m-2s- } \\
1\end{array}$ & $\mathrm{R}$ & $\mathrm{W}$ & $\begin{array}{l}\mathrm{Vm} / 10-1 \\
\mathrm{~m} 3 \text { mole-1 }\end{array}$ & $\begin{array}{l}\text { Vf / 10-6 } \\
\text { m3mole-1 }\end{array}$ \\
\hline \multicolumn{10}{|c|}{$0.2 \%$ PVA - Zn. St. - EG } \\
\hline 0.01 & 1530 & 1086.6 & 1.29 & 3.93 & 1.66 & 9.03 & 17.28 & 0.78 & 3.62 \\
\hline 0.02 & 1532 & 1087.2 & 1.31 & 3.92 & 1.67 & 9.03 & 17.29 & 0.78 & 3.55 \\
\hline 0.03 & 1548 & 1087.8 & 1.33 & 3.84 & 1.68 & 9.06 & 17.34 & 0.78 & 3.53 \\
\hline 0.04 & 1568 & 1088.5 & 1.35 & 3.74 & 1.71 & 9.10 & 17.41 & 0.78 & 3.53 \\
\hline 0.05 & 1570 & 1089.1 & 1.37 & 3.72 & 1.71 & 9.10 & 17.41 & 0.78 & 3.47 \\
\hline 0.06 & 1576 & 1089.7 & 1.40 & 3.69 & 1.72 & 9.11 & 17.43 & 0.78 & 3.36 \\
\hline 0.07 & 1584 & 1090.4 & 1.44 & 3.66 & 1.73 & 9.13 & 17.46 & 0.78 & 3.26 \\
\hline 0.08 & 1600 & 1091.0 & 1.46 & 3.58 & 1.75 & 9.16 & 17.51 & 0.78 & 3.25 \\
\hline 0.09 & 1608 & 1091.6 & 1.49 & 3.54 & 1.76 & 9.17 & 17.53 & 0.78 & 3.15 \\
\hline 0.1 & 1618 & 1092.3 & 1.57 & 3.50 & 1.77 & 9.19 & 17.56 & 0.78 & 2.97 \\
\hline \multicolumn{10}{|c|}{$0.2 \%$ PVA - Ca. St. - EG } \\
\hline 0.01 & 1532 & 1086.6 & 1.34 & 3.92 & 1.66 & 9.03 & 17.29 & 0.78 & 3.41 \\
\hline 0.02 & 1556 & 1087.2 & 1.36 & 3.80 & 1.69 & 9.08 & 17.37 & 0.78 & 3.42 \\
\hline 0.03 & 1568 & 1087.8 & 1.40 & 3.74 & 1.71 & 9.10 & 17.41 & 0.78 & 3.33 \\
\hline 0.04 & 1576 & 1088.4 & 1.45 & 3.70 & 1.72 & 9.12 & 17.43 & 0.78 & 3.18 \\
\hline 0.05 & 1588 & 1089.0 & 1.51 & 3.64 & 1.73 & 9.14 & 17.47 & 0.78 & 3.04 \\
\hline 0.06 & 1596 & 1089.6 & 1.54 & 3.60 & 1.74 & 9.15 & 17.50 & 0.78 & 2.96 \\
\hline 0.07 & 1600 & 1090.2 & 1.58 & 3.58 & 1.74 & 9.16 & 17.51 & 0.78 & 2.87 \\
\hline 0.08 & 1610 & 1090.8 & 1.60 & 3.54 & 1.76 & 9.18 & 17.54 & 0.78 & 2.85 \\
\hline 0.09 & 1620 & 1091.4 & 1.65 & 3.49 & 1.77 & 9.20 & 17.57 & 0.78 & 2.74 \\
\hline 0.1 & 1634 & 1092.0 & 1.69 & 3.43 & 1.78 & 9.22 & 17.61 & 0.78 & 2.69 \\
\hline
\end{tabular}

Table 3 : Thermodynamic parameters of ternary mixtures at $303 \mathrm{~K}$

\begin{tabular}{llllll}
\hline $\begin{array}{l}\text { Conc of } \\
\text { stearate }\end{array}$ & $\begin{array}{l}\mathrm{Lf} / 10-11 \\
\mathrm{M}\end{array}$ & $\begin{array}{l}\pi / 105 \\
\mathrm{~atm}\end{array}$ & $\begin{array}{l}\alpha / \mathrm{f} 2 / 10-11 \\
\mathrm{~m}-1 \mathrm{~s} 2\end{array}$ & $\mathrm{i} / 10-9$ & $\mathrm{~s}$ \\
$0.2 \mathrm{PVA}-\mathrm{Zn}$. St. $-\mathrm{EG}$ & & & & $\mathrm{m}$ \\
0.02 & 3.93 & 3.86 & 0.873 & 0.677 & 8.65 \\
0.03 & 3.93 & 3.89 & 0.881 & 0.684 & 8.68 \\
0.04 & 3.89 & 3.89 & 0.866 & 0.679 & 8.88 \\
0.05 & 3.84 & 3.89 & 0.844 & 0.671 & 9.13 \\
0.06 & 3.83 & 3.92 & 0.852 & 0.678 & 9.16 \\
0.07 & 3.81 & 3.96 & 0.864 & 0.690 & 9.24 \\
0.08 & 3.79 & 4.00 & 0.872 & 0.701 & 9.34 \\
0.09 & 3.75 & 4.00 & 0.857 & 0.695 & 9.55 \\
0.1 & 3.73 & 4.04 & 0.865 & 0.705 & 9.65 \\
$0.2 \% \mathrm{PVA}-\mathrm{Ca}$ & 3.71 & 4.13 & 0.889 & 0.730 & 9.78 \\
0.01 & 3.930 & & & & \\
0.02 & 3.870 & 3.940 & 0.9050 & 0.7030 & 8.68 \\
0.03 & 3.840 & 3.930 & 0.8750 & 0.6900 & 8.98 \\
0.04 & 3.820 & 3.970 & 0.8770 & 0.6980 & 9.14 \\
0.05 & 3.790 & 4.030 & 0.8970 & 0.7170 & 9.24 \\
0.06 & 3.770 & 4.130 & 0.9090 & 0.7320 & 9.39 \\
0.07 & 3.760 & 4.170 & 0.9160 & 0.7410 & 9.50 \\
0.08 & 3.730 & 4.180 & 0.9300 & 0.7550 & 9.55 \\
0.09 & 3.710 & 4.240 & 0.9230 & 0.7540 & 9.68 \\
0.1 & 3.670 & 4.270 & 0.9360 & 0.7690 & 9.81 \\
\hline & & & & & 10.00 \\
\hline
\end{tabular}


Table 4 : Excess parameters of ternary mixtures like UE, BE, ZE,LfE and vmE at $303 \mathrm{~K}$

\begin{tabular}{|c|c|c|c|c|c|}
\hline Conc of stearate & $\begin{array}{l}\text { UE } \\
\text { ms-1 }\end{array}$ & $\begin{array}{l}\beta E / 10-11 \\
\mathrm{Kg}-1 \mathrm{~ms}-2\end{array}$ & $\begin{array}{l}\mathrm{ZE} / 105 \\
\mathrm{Kg} \text { m-2s-1 }\end{array}$ & $\begin{array}{l}\text { LfE / 10-12 } \\
\text { m }\end{array}$ & $\begin{array}{c}\text { VmE / 10-3 } \\
\text { m3mole-1 }\end{array}$ \\
\hline \multicolumn{6}{|c|}{$0.2 \%$ PVA - Zn. St. - EG } \\
\hline 0.01 & -106 & 0.4640 & 8.940 & 0.2330 & -0.4410 \\
\hline 0.02 & -104 & 0.3330 & 9.250 & 0.1680 & -0.8700 \\
\hline 0.03 & -88 & -0.5000 & 11.10 & -0.2520 & -1.300 \\
\hline 0.04 & -68 & -1.500 & 13.40 & -0.7610 & -1.730 \\
\hline 0.05 & -66 & -1.620 & 13.70 & -0.8240 & -2.160 \\
\hline 0.06 & -59 & -1.930 & 14.40 & -0.9830 & -2.580 \\
\hline 0.07 & -51 & -2.330 & 15.40 & -1.190 & -3.010 \\
\hline 0.08 & -35 & -3.080 & 17.20 & -1.580 & -3.440 \\
\hline 0.09 & -27 & -3.460 & 18.20 & -1.780 & -3.870 \\
\hline 0.1 & -17 & -3.920 & 19.40 & -2.030 & -4.300 \\
\hline \multicolumn{6}{|c|}{$0.2 \%$ PVA - Ca. St. - EG } \\
\hline 0.01 & -104 & 0.3570 & 0.9160 & 0.1800 & -0.4210 \\
\hline 0.02 & -80 & -0.8740 & 1.190 & -0.4420 & -0.8290 \\
\hline 0.03 & -68 & -1.480 & 1.330 & -0.7530 & -1.240 \\
\hline 0.04 & -59 & -1.890 & 1.420 & -0.9630 & -1.640 \\
\hline 0.05 & -47 & -2.480 & 1.560 & -1.270 & -2.050 \\
\hline 0.06 & -39 & -2.870 & 1.660 & -1.470 & -2.460 \\
\hline 0.07 & -35 & -3.080 & 1.720 & -1.580 & -2.870 \\
\hline 0.08 & -25 & -3.560 & 1.830 & -1.830 & -3.280 \\
\hline 0.09 & -14 & -4.020 & 1.950 & -2.070 & -3.680 \\
\hline 0.1 & 0 & -4.640 & 2.120 & -2.410 & -4.090 \\
\hline
\end{tabular}

Table 5 Experimental velocities and theoretical velocities along with the percentage deviation of ternary mixtures at $303 \mathrm{~K}$

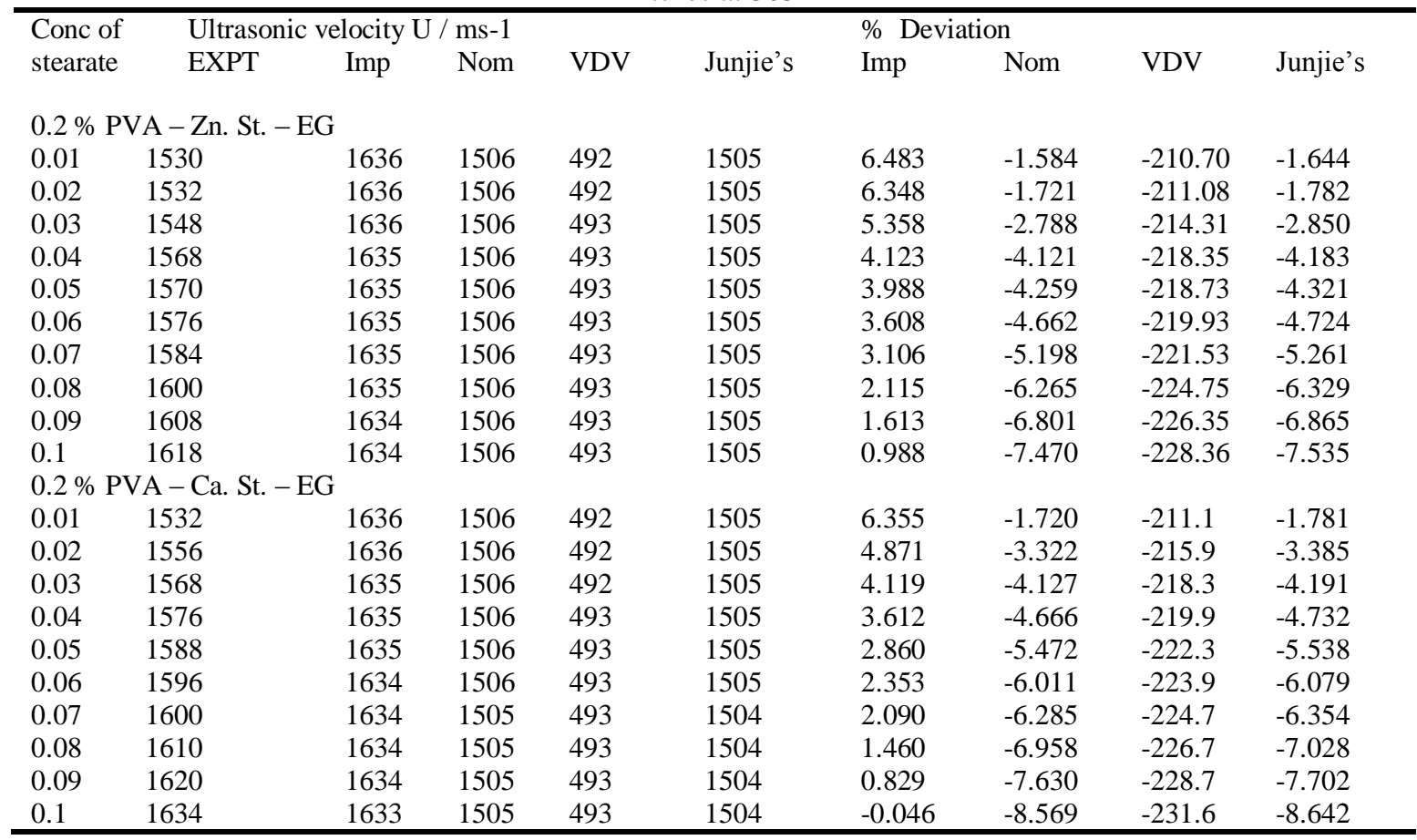



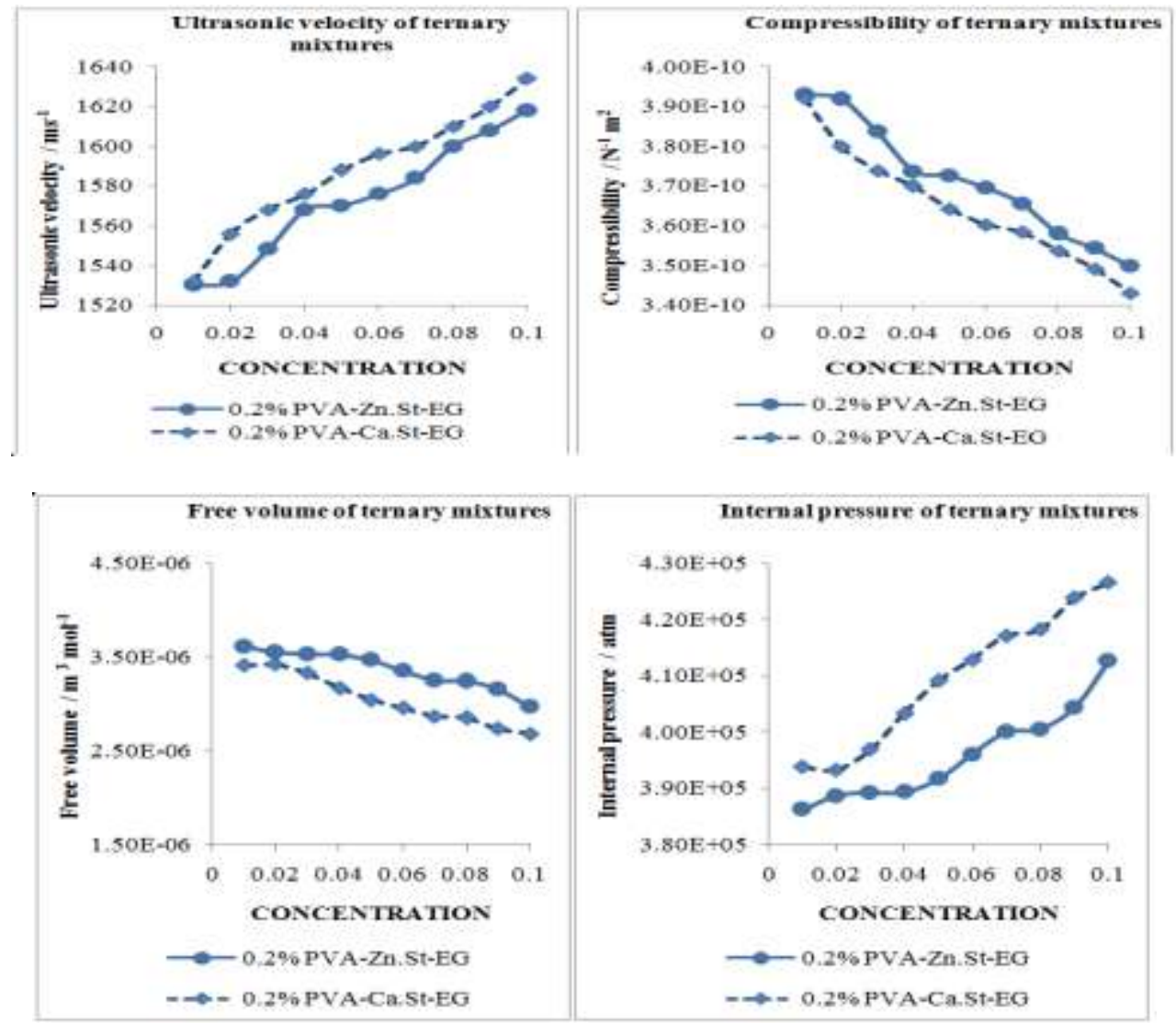

Fig. 1 : Computed parameters of $0.2 \%$ PVA - Zn. St - EG and $0.2 \%$ PVA - Ca. St - EG systems
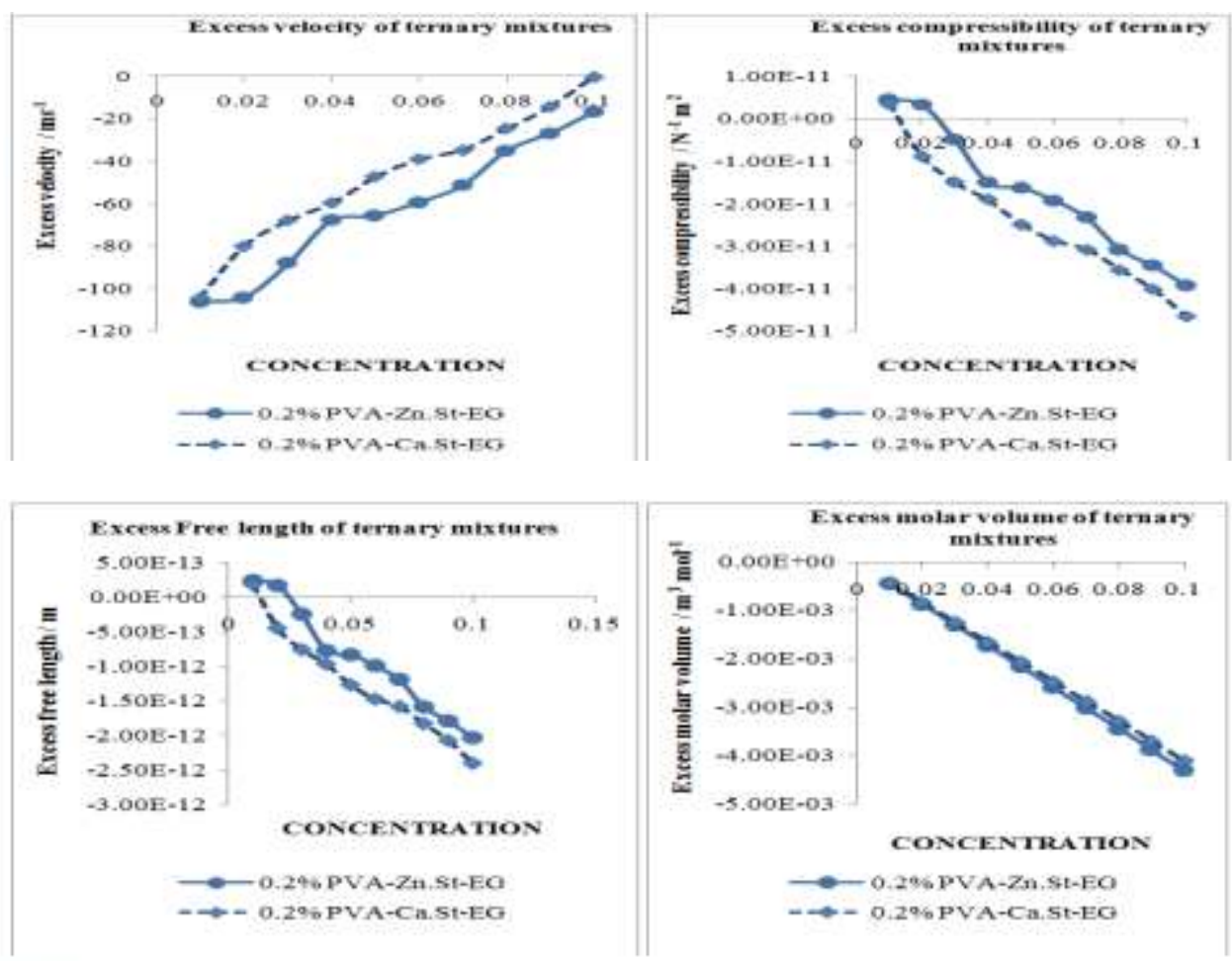

Fis.2: Excess paraneters of $0.2 \%$ PVA $-\mathrm{Zn}$. $\mathrm{st}-\mathrm{EO}$ and $0.2 \% \mathrm{PVA}-\mathrm{Ca}$. St $-\mathrm{EO}$ syntems

In the present investigation, studies are made with different $0.2 \%$ polyvinyl alcohol concentration with two different stabilizers zinc stearate and calcium stearate. Ethylene glycol is used as a plasticizer for the entire composition range. Ternary mixture is prepared using polyvinyl alcohol, stearate and ethylene glycol of different concentration and ultrasonic investigation is carried out. Polyvinyl alcohol unlike many polymers, is 
soluble in water. Ultrasonic methods have been used for the mechanical characterization of hydrogels by several researchers [3]. In the blends, the presence of chemically grafted units is of major importance in providing adhesion between the polymers. Interesting products can be formed from some polymers even without the requirement of a chemical graft, in such cases weaker secondary forces such as hydrogen bonds play a dominant part. Many experimental and theoretical methods have been used to investigate polymer - polymer compatibility and also the interactions between polymers [4]. Ultrasonic velocity, density and viscometric measurements may reveal various aspects of the compatibility of polymer blends in highly viscous or solid forms. It also pointed out that ultrasonic velocity varies linearly with composition in compatible polymer blends and nonlinearly in semicompatible and incompatible blends. Hence a combination of methods is generally used. Polyvinyl alcohol is used as an excellent film forming, emulsifying and adhesive properties. The water, which acts as a plasticiser, will then reduce its tensile strength, but increases its elongation and tear strength. PVA is fully degradable and is a quick dissolver. PVA has a melting point of $230^{\circ} \mathrm{C}$ and $180-190^{\circ} \mathrm{C}$ for the fully hydrolysed and partially hydrolysed grades, respectively. It decomposes rapidly above $200^{\circ} \mathrm{C}$ as it can undergo pyrolysis at high temperatures.

It can be that the ultrasonic velocity (U) is found to increase with increase in stearate concentration. Polyvinyl alcohol exhibits polyelectrolyte properties in solution, a flexible non - ionic polymer which obeys the classical Huggin's equation. It may be attributed to the mutual attraction of macromolecules in solution because of the increase of hydrodynamic and thermodynamic interaction. Linear variation in ultrasonic velocity for all concentration range reveals that greater specific interaction exists between polymer segments which may be due to hydrogen bonding. It also confirms that the solution forms miscible blend [5]. The force of attraction of polar groups across the solvating molecules provides scopes for further association leading to solute - solvent interaction [6]. Ethylene glycol is similar to water form 3D network of hydrogen bonds, strong association of glycol molecules in a liquid state is the reason of its high relatively high temperature of melting point and that of boiling point. Ethylene glycol is bidentate ligand when forming complex with transition metals [7]. Glycol molecules involved in first coordination sphere are additionally hydrogen bonded. Ethylene glycol is too small to be bent enough to form two coordination bonding with cation, such as zinc or calcium of stearate used in the present study. As ionic radius is large, it forms ionic pairs that are separated by solvent or hydrogen bonded to the network of ethylene glycol in the bulk [8]. It predicts stronger attraction between polymer, stearate and ethylene glycol by means of strong hydrogen bond. Comparing stearates, zinc stearate and calcium stearate shows only slight variation in polymer solution. The density values $(\rho)$ increases with increase in stearate concentration and viscosity values $(\eta)$ increases with increase in stearate that reveals the existence of intermolecular hydrogen bonding. Increase in viscosity predicts that molecules become less mobile in solution and velocity gradients around the collapsing bubbles to therefore become smaller, resulting in lower extents of viscosity reduction.

The adiabatic compressibility $(\beta)$ decreases with increase in stearate concentration. Hydration effect decreases adiabatic compressibility which confirms the presence of strong solute - solvent interactions through dipole - dipole interactions of the hydroxyl group of glycol with water molecules [9]. A positive value of adiabatic compressibility shows weakening of the network of ethylene glycol molecules, hydrophobic when interactions with water. Hydrogen bonding between ethylene glycol elements with polyvinyl alcohol and stearate inside the network shows similar bonding could exist in liquid non - polymerized diols. The structure breaking effects is conserved in ethylene glycol, suggesting hydrophobicity of the solute - solvent interactions. This confirms the assumption expressed in introduction that water in polyvinyl alcohol solution and ethylene glycol are structurally similar and the process occurring when adding either zinc stearate or calcium stearate to them can be interpreted in similar manner. One or two ethylene glycol molecules form bridges linking either zinc or calcium ion, other glycols coordinate to this system in this typical way. Comparatively in addition of either zinc stearate or calcium stearate, similar decrease in trend is observed in all concentration range. Acoustic impedance $(Z)$ increases linearly in addition of either zinc stearate or calcium stearate confirms the presence of molecular association between solute - solvent molecules through intermolecular hydrogen bonding. Rao's constant (R) and Wada's constant (W) increases linearly with increase in stearate concentration with ethylene glycol, indicates the presence of solute - solvent interaction and confirms the absence of complex formation [10]. Molar volume ( $\mathrm{Vm}$ )shows constant value for entire stearate concentration range. The variation of free volume (Vf) decreases with increase in stearate concentration for $0.2 \% \mathrm{PVA}-\mathrm{Zn}$. St $-\mathrm{EG}$ systems and non linear variation is observed for $0.2 \%$ of PVA $-\mathrm{Ca}$. St - EG system.

The intermolecular free length (Lf) also follows the same trend as that of adiabatic compressibility, the decrease in free length with increase in stearate concentration reveals the presence of strong interaction. According to Eyring liquid state theory, the acoustic wave which was excited in the fluid is transmitted momentarily to the intermolecular free length in the molecule having the spherical or symmetrical shape is short leading to a high speed of sound [11]. Internal pressure values $(\pi \mathrm{i})$ increases with increase in stearate concentration for $0.2 \% \mathrm{PVA}-\mathrm{Zn}$. St $-\mathrm{EG}$ systems. The reverse trend is observed for $0.2 \% \mathrm{PVA}-\mathrm{Ca}$. St $-\mathrm{EG}$ 
systems. Increase in internal pressure indicates association through hydrogen bonding suggesting the close packing of the molecules inside the shield, which may be brought about by the increasing magnitude of interaction [12]. The absorption coefficient values $(\alpha / \mathrm{f} 2)$ shows non linear variation, It increase and decrease with increase in stearate concentration for $0.2 \% \mathrm{PVA}-\mathrm{Zn}$. St - EG systems. Comparatively, $0.2 \%$ PVA - Ca. $\mathrm{St}$ - EG systems, reverse trend is observed. In addition of either zinc stearate or calcium stearate to polyvinyl alcohol and ethylene glycol, calcium stearate shows greater absorption coefficient than zinc stearate. The values of relaxation time are calculated $(\tau)$ shows non linear variation for both the systems. For $0.2 \% \mathrm{PVA}-\mathrm{Zn}$. St EG systems, it increases and decreases with increase in stearate concentration. The reverse trend is observed $0.2 \%$ PVA - Ca. St - EG systems. The interaction parameter value $(\alpha)$ increases with increase in stearate concentration [13]. Intermolecular attraction signifies that unlike interactions are relatively strong compared to like interactions. Comparatively addition of calcium stearate with polyvinyl alcohol and ethylene glycol shows greater $\alpha$ value compared than zinc stearate.

According to Fort and Moore, excess values are negative of unequal molecular size in which dispersion force are dominant but experimental data collected in the literature upto the present provide strong evidence that for mixture in which strong attractive interaction such as dipole - dipole, dipole - induced dipole, hydrogen bonding are likely to occur between the components [14]. The excess velocity shows negative deviation for 0.2 $\%$ PVA - Zn. St - EG systems, it shows positive deviation for $0.2 \%$ PVA - Ca. St - EG systems. Negative deviation shows that dissociation of individual molecule and association of molecule by increasing rate of dissociation of like molecules. It may reveal the rupture of cohesion and growing adhesion between the components polyvinyl alcohol, stearates and ethylene glycol leading to stronger molecular interaction. Positive deviation of excess ultrasonic velocity confirms the existence of weak interaction. The presence of strong hydrogen bonding interactions between unlike molecules indicates negative deviation in excess parameters of adiabatic compressibility ( $\beta$ E) [15]. In $0.2 \%$ of PVA - Zn. St - EG system and PVA - Ca. St - EG system, excess adiabatic compressibility shows positive value at lower stearate concentration that indicates the presence of dispersive interactions. Positive deviation of compressibility from linear value suggests the presence of very low cohesive energy among the molecules which is possible only when two molecules combine together to form a single unit. In other words, in all these mixtures due to hydrogen bond formation predominates over the dipole association. As stearate concentration increases, it shows negative values that confirms greater molecular interaction among polyvinyl alcohol, stearate and ethylene glycol. The excess free length (LfE) also follows the same trend as that of excess adiabatic compressibility predicting that negative deviation shows the existence of strong interaction and positive deviation shows the presence of weak interaction. It was reported that the positive deviation is observed in excess impedance (ZE) indicates that interstitial accommodation [16] does not exist and it leads to strong molecular interaction between molecules through intermolecular hydrogen bonding. The excess molar volume (VME) is found to be negative for entire composition range with increase in stearate concentration. Excess molar volume arises due to the presence of difference in intermolecular interaction energy between like and unlike molecules, difference in size and shape of components, any structural changes on addition of second component, formation of new chemical species through hydrogen bonding or electron donor - acceptor interactions. Negative deviation explains the self dissociation and physical dipole - dipole interaction between the components which increases disorder of the breaking of ordered structure, predominates the loss in free volume. The sign reflects superposition of size effect upon another which causes contraction that may arise from disruption by mixing process of expanded dipole - dipole attraction. The restriction in rotational motion which arises when straight chain component liquid are accommodated interstitially within the ring structured.

It can be seen that the theoretical values of ultrasonic velocity computed by four theories shows deviation from experimental values. On mixing two or more components, molecular interaction takes place due to the presence of various chemical forces between solute and solvent molecules. Theoretical evaluation of ultrasonic velocity shows that the percentage variations is quite less in impedance relation, Nomoto's relation, Junjie relation and maximum negative deviation in Van Dael and Vangeel's relation. In all polymer concentration with increase in concentration of either zinc stearate or calcium stearate with ethylene glycol shows maximum negative deviation of roughly 200 in Van Dael and Vangeel relation. It reveals the presence of inter molecular hydrogen bonding in the mixture, between the hydroxyl group of ethylene glycol and PVA molecule incorporating either the zinc or calcium ion of stearate molecule in it.

\section{Conclusion}

In the present investigation, $0.2 \%$ polyvinyl alcohol solutions were employed along with either zinc stearate or calcium stearate and ethylene glycol. A systematic study of ternary mixtures of polyvinyl alcohol, stearate and ethylene glycol has been carried out at different stearate concentrations at $303 \mathrm{~K}$. The aim of this investigation was to correlate this finding with liquid - solid - liquid mixtures. According to the results obtained, structure breaking character of ethylene glycol, too suggesting hydrophobicity of solute - solvent interactions causing destruction of water in polyvinyl alcohol, the broken water molecules enter the vacant space 
of EG and thus get trapped. It reveals the presence of strong solute - solvent interaction due to hydrogen bonding between ethylene glycol and polyvinyl alcohol, by trapping the metal cation of stearate within them leading to compact structure. Thus locally the structure becomes more open frame work. This confirms the assumption expressed in introduction that water in polyvinyl alcohol and ethylene glycol are structurally similar and the processes occurring when adding stearate to them can be interpreted in a similar manner. Comparing the effect of addition of stearates to polymer and plasticizer, calcium stearate shows maximum molecular interaction than zinc stearate. It is evident from the calculated negative excess values in $\mathrm{UE}, \beta \mathrm{E}, \mathrm{Lf} \mathrm{E}, \mathrm{VmE}$ and positive values in $\mathrm{ZE}$, that strong intermolecular hydrogen bonding exists between polyvinyl alcohol, stearate and ethylene glycol with increase in polymer and stearate concentration. From the computed theoretical velocity calculations, least deviation is found in Impedance, Nomoto, Junjie relation and maximum negative deviation is observed in Van Dael and Vangeel relation which confirms the existence of strong intermolecular hydrogen bonding.

\section{References}

[1] Madras G, Chattopadhyay S Polym. Degrad. Stab. 73 (2001) 33

[2] Tompa H Polymer solutions, London 1956

[3] Price G J, West P J, Smith P F Ultrason. Sonochem, 1 (1994) 51.

[4] G.J. Price, P.F. SmithPolymer 34 (1993) 4111.

[5] J. Chakraborty, J. Sarkar, R. Kumar, G. Madras Polym. Degrad. Stab. 85 (2004) 555.

[6] A. Akyuz, H. Catalgil-Giz, A.T. GizMacromol. Chem. Phys. 209 (2008) 801.

[7] Palladhi, R., \& Singh, R. P Journal of Applied Polymer Science, 51, (1994a) 1559.

[8] W. Schnable Polymer degradation, Hanser, New York, USA, 1981.

[9] Mikkonen R and Savlainen A J. Appl. Polym. Sci., 39 (1990) 1709.

[10] Peter Hauptmann, Ralph Sauberlich and Klaus Schlothauer Polymer, vol 25 (1984) 985

[11] G.J. Price, P.F. Smith Eur. Polym. J. 29 (1993) 419.

[12] R Kumar, S Jayakumar and V Kannappan., Indian Journal of Pure \& Applied Physics., Vol. 46. March 2008, pp. 169 - 175.

[13] Ulagendran, R Kumar, S Jayakumar and V Kannappan., Journal of Molecular liquids., 148 (2009) pp. 67 - 72.

[14] R Kavitha, S Jayakumar and R Uma ISST Journal of Applied Chemistry, Vol.1, No.2 (2010) 57.

[15] R Kavitha, S Jayakumar and R Uma IJESR, Vol.2, Issue 4 (2011) 293.

[16] R Kavitha, S Jayakumar and R Uma International Journal of Chemistry and Applications, Vol.3, No.1 (2011) 19. 